\title{
Kinked Tail
}

National Cancer Institute

\section{Source}

National Cancer Institute. Kinked Tail. NCI Thesaurus. Code C124552.

A finding that the tail has a localized undulation. 